\title{
S and C Bands Multilayer T-Slot Photonic Band gap Micro Strip Antenna
}

\author{
Purvai Rastogi \\ ME student JEC Jabalpur \\ Kanchan Cecil \\ Asst. Prof JEC Jabalpur
}

\begin{abstract}
A design strategy using Photonic Band Gap (PBG) structure on patch to achieve wider bandwidth for patch antenna is presented in this paper. It is found that, the impedance bandwidth has improved from $30 \%$ to $50 \%$ at centre frequency $2 \mathrm{GHz}$ after adding PBG on the patch. The antenna has dual band operations at 2 and 6 GHz. Antenna's whole geometry is consist of a three layer (glass epoxy - air - glass epoxy).We have also studied the effects of different parameters of antenna in finite ground plane. All results validated in IE3D ${ }^{\mathrm{TM}}$ Simulator. This proposed antenna is used for satellite, and wireless communication at, S Band and C Band.
\end{abstract}

Keywords: IE3D ${ }^{\mathrm{TM}}$ Simulator, Micro strips Antenna, T- slot, Photonic Band Gap, S- C Band.

\section{Introduction}

An explosive growth of the wireless radio communication systems is currently observed in the microwave band. In the short range communications or contactless identification systems, antennas are key components, which must be small, low profile, and with minimal processing costs. The main limitations of the Micro-strip antennas are low gain and narrow impedance bandwidth. The bandwidth of the Micro-strip antenna can be increased using various techniques such as by loading a patch, by using a thicker substrate, by reducing the dielectric constant, by using gap-coupled multi-resonator etc. However, using a thicker substrate causes generation of spurious radiation and there are some practical problems in decreasing the dielectric constant. The spurious radiation degrades the antenna parameters. Among various antenna bandwidth enhancement configurations, the two gap-coupled Circular Microstrips patch antenna is most elegant one. So, gapcoupling is the suitable method for enhancing the impedance bandwidth of the antennas. In the configuration of gap-coupled Micro-strip antennas method, two patches are placed close to each other. The gap-coupled Micro-strip antennas generate two resonant frequencies and the bandwidth of the Micro-strip antennas can be increased. Rectangular Micro-strip patch antenna with multiband frequency operation is designed by slits loaded on active and parasitic patch the results are investigated. High gain of operation achieved due to gap between itself parasitic patch, gap between parasitic patch, and active patch. We investigated hybrid modeling for proposed Micro-strip antenna. We investigated spacing between active and parasitic patch, spacing and height between parasitic patch by using iterative method on IE3D ${ }^{\mathrm{TM}}$ Simulator (MOM Simulator).We studied enhances the bandwidth and reducing loss. The microstrip antenna "many of researches have been made on exploring more efficient antenna".

The use of Photonic Band Gap (PBG) structure is becoming attractive for many researchers in electromagnetic and antenna field. PBG had been used to improve the performance of various antennas such as patch antenna and resonant antenna. Micro strip patch antenna is promising to be a good candidate for future wireless technologies. Micro strip patch antenna consists of a dielectric substrate, with a ground plane on the other side. Due to its advantages such as low weight, low profile planar configuration, low fabrication costs and capability to integrate with microwave integrated circuits technology, the micro strip patch antenna is very well suited for applications such as wireless communications system, cellular phones, pagers, Radar systems and satellite communications systems.

In this paper, a compact dual band design of Micro strip patch antenna using a PBG slot on the patch is proposed. The bandwidth has improved by adding the PBG T slots.

1. Proposed geometry design Analysis

Proposed Rectangular Micro-strip antenna Included

a. Six T- slot loaded on top patch

b. $\Delta=10$ mil Air gap between layer

c. Whole geometry consist by thee layer glass epoxy PCB and air gap(FR-4 - air- FR-4)

d. Total height of geometry is 128mil (from ground plan to top layer)

e. Top layer consists of patch dimension $\mathrm{Lx}$ W= $800 \times 900 \mathrm{mil}^{2}$

f. Ground plan have dimension $\mathrm{L} x \mathrm{~W}=1568 \mathrm{x}$ 1668 mil $^{2}$ 


\section{Finite ground plane}

Specification of Glass epoxy PCB is $\varepsilon_{\mathrm{r}}=4.3, \mathrm{~h}=59 \mathrm{n}$ and loss tangent $\tan \delta=.019$, we have substituted six slot of different dimensions on the top layer patch. T proposed model shown in figure- 2 and top view shov in figure-1

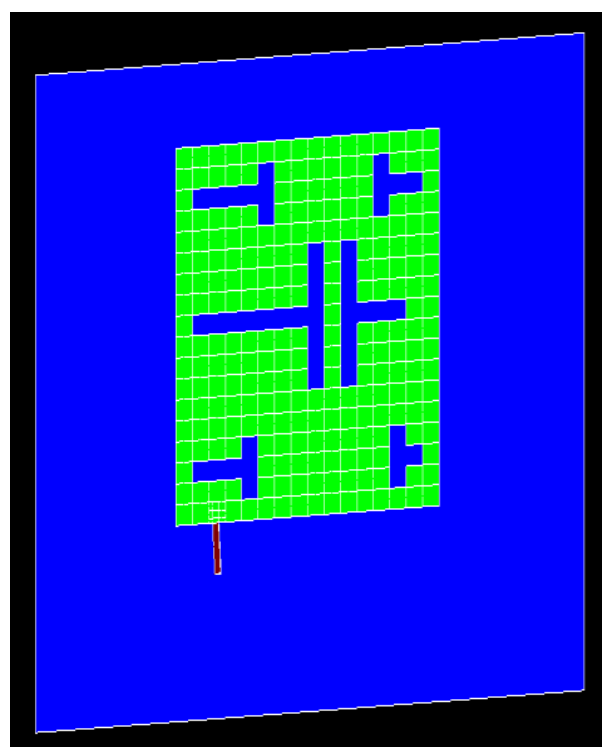

Fig 1 Top View for Proposed model

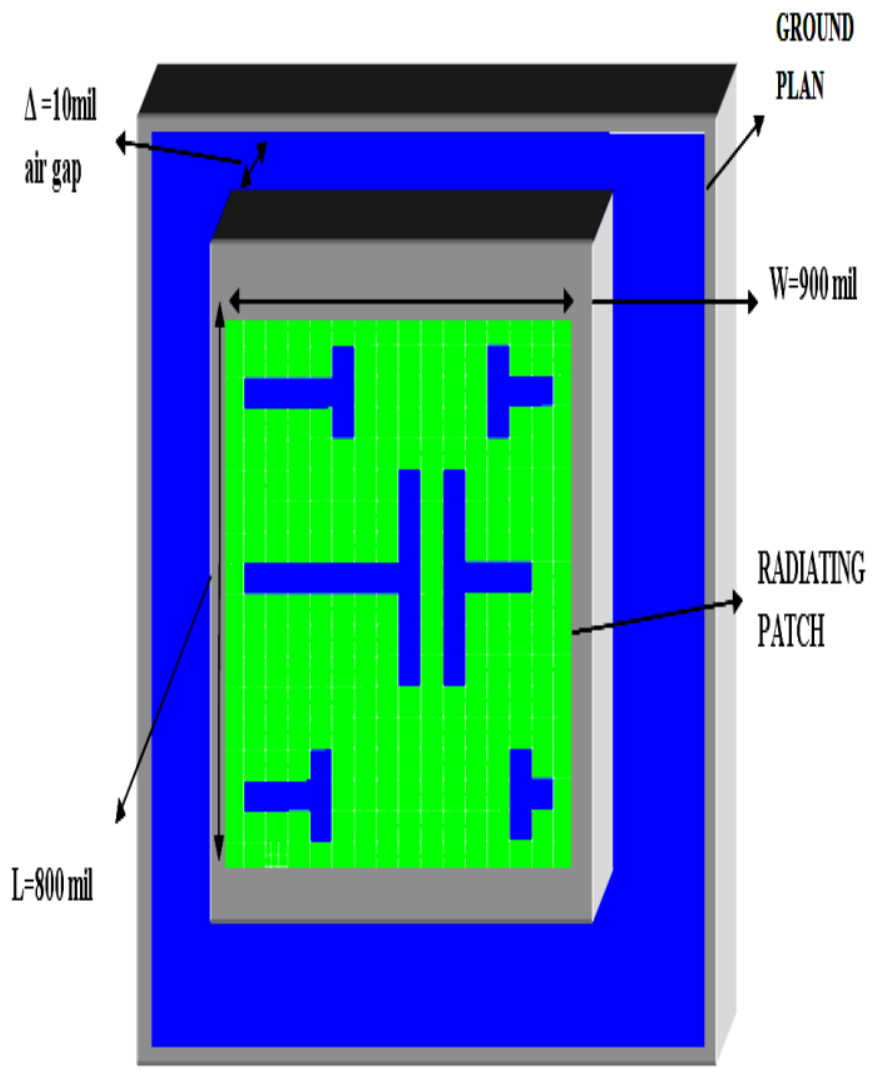

Fig 2 Proposed design model

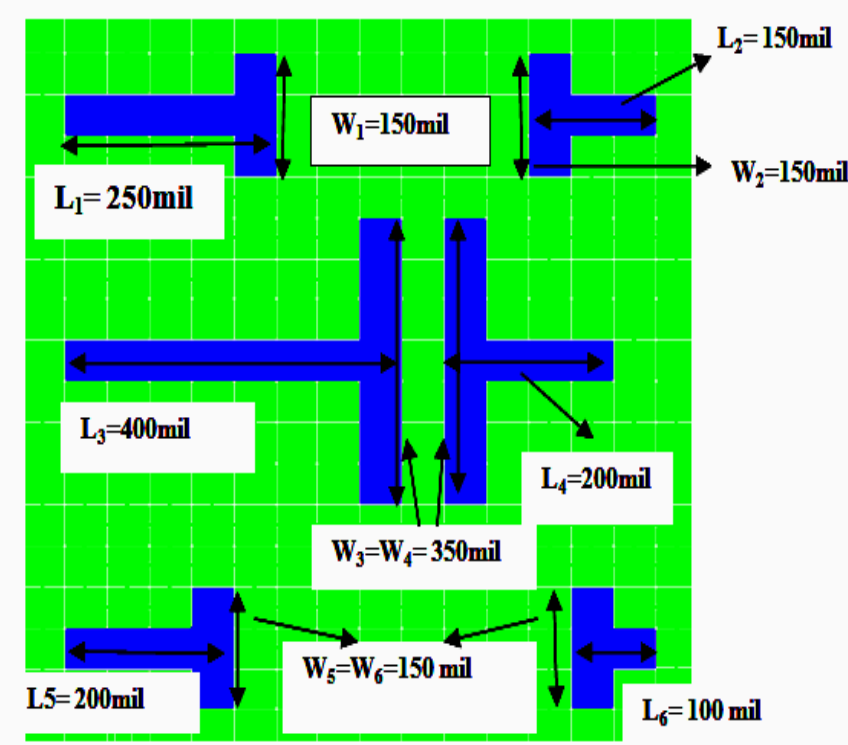

Figure 3 Dimension of the Top Patch

\section{Result and discussion}

This modeling used for improvement in overall performance of Micro-strip antenna. We have focused on optimal gain and dual band. For achieving these outcomes, genetic algorithm method on IE3D ${ }^{\mathrm{TM}}$ Simulator. The impedance frequency bandwidth of a Micro-strip antenna depends primarily on both the thickness and the dielectric permittivity of the substrate. A thick substrate with a low dielectric permittivity can increase the bandwidth of the printed patch. The technique of stacked patches is based on the fact that bandwidth is in general proportional to the antenna volume measured in wavelengths but at the same time a relative large volume is a disadvantage for many applications. The utilization of additional parasitic patches of different patches of different size directly -or- gap coupled with main patch is an effective method. Superior to these methods is the techniques of slot loading or texturing the patches by slits because they ensure the small size and the low profile of the antennas. The wideband performance of the slit loaded patch is based similarly to the method of slot loading, on the excitation of more than one adjacent resonant mode. Moreover the presence of the slits in the vicinity of the feeding probe could add a capacitive load at the input impedance of the patch. This capacitive load could effectively contribute to the resonance of the patch because can counteract the inductive part of the probe's input impedance. It is noticed that this inductive part would inevitably be arge if a thick substrate is chosen for wideband operation so the insertion of slits enhances by two ways the width of the operation band, and it has been 
reported greater bandwidth. The width of the frequency band of the antenna can be controlled by slits' length and width and the slits' position. The slits divided the in more parts and each one corresponding an equivalent circuit of resonance. We achieved good impedance matching due to all aspect of modeling.

\subsection{Return Loss vs. frequency}

After IE3D ${ }^{\mathrm{TM}}$ Simulation more coupling between patches so that overall loss due to surface wave, cross polarization and poor impedance matching have been reduced. We observed self and mutual impedance effect between active and parasitic patch, between in top (128mil), these self impedance and mutual impedance provides good impedance matching over broad band. All result shown figure-3 and Table-1.

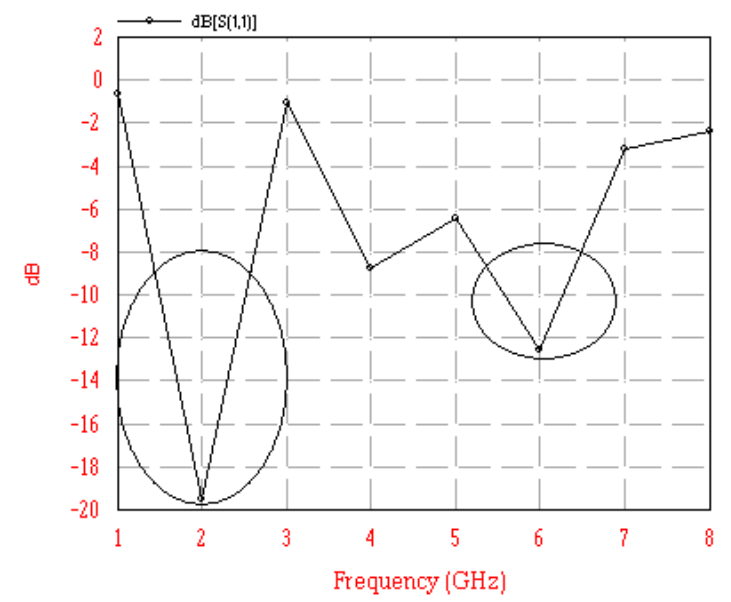

Figure 3 Return Loss vs. frequency

\subsection{VSWR vs. Frequency}

We have simulated proposed geometry and simulated VSWR result shown in figure-4, and Result Table.

\begin{tabular}{|c|c|c|c|}
\hline $\begin{array}{c}\text { Frequency } \\
(\mathrm{GHz})\end{array}$ & VSWR & $\begin{array}{c}\text { Return } \\
\text { Loss } \\
(\mathrm{dBi})\end{array}$ & $\begin{array}{c}\text { Maximum } \\
\text { Directive } \\
\text { Gain (dBi) }\end{array}$ \\
\hline 2 & 1.103 & -19.5 & 3.5 \\
\hline 6 & 1.6 & -12 & 5.5 \\
\hline
\end{tabular}

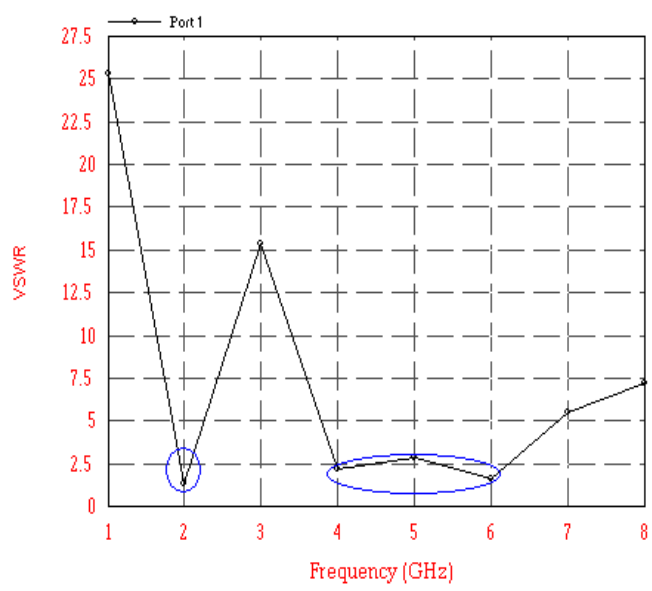

\subsection{Smith chart Analysis}

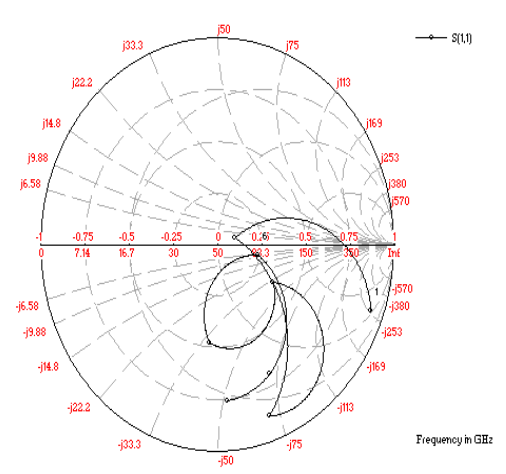

Fig : 5

\subsection{Maximum Directional Gain analysis}

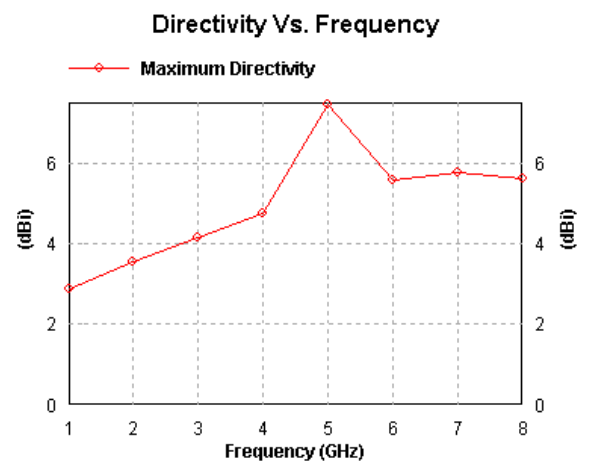

Figure 5 Directivity vs. frequency

\subsection{Simulation Result Table}

The results of proposed Micro-strip antenna. Simulated on IE3D ${ }^{\mathrm{TM}}$ Simulator, Investigated results of VSWR, Directional Gain and return loss shown in Result Table :- 


\subsection{Radiation Pattern}

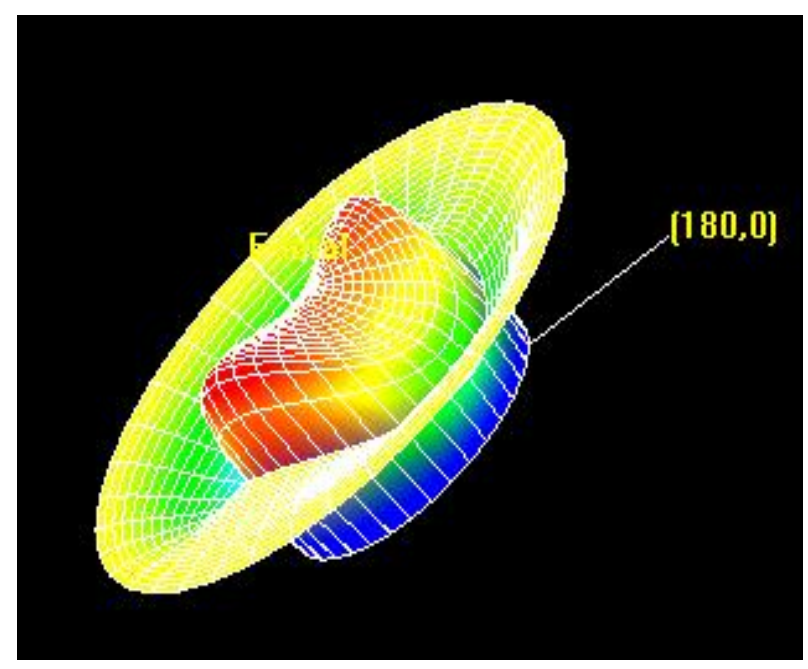

Fig : 6

\section{Conclusions}

In this paper, Photonic band gap T-slot loading on multilayer Micro-strip antenna is presented. We studied broadband frequency of operation demonstrated by single geometry. We have achieved dual band $51 \%$ bandwidth(at $2 \mathrm{GHz}$ center frequency), $11 \%$ Bandwidth (at $6 \mathrm{GHz}$ center frequency) and maximum directional gain up to $7 \mathrm{dBi}$ by using gap-coupled multiresonator loaded on active patch; appropriate configurations for the presented antenna are suggested. The simulated results are presented and discussed for appropriate geometry on IE3D ${ }^{\mathrm{TM}}$ SIMULATOR by genetic algorithm method. The stack geometry of a single probe fed rectangular Micro-strip antenna incorporating a slot loaded and gap coupled with parasitic and active patch is studied. After loading a slot multi resonator dual band operation achieved. We investigated enhancement in maximum directional gain by using concept of stack geometry with one active and multiple parasitic patch of different dimensions. This proposed antenna is used for satellite, Radar, and wireless communication S-Band and C Band.

\section{References}

[1] M. Mahdi Honari, Abdolali Abdipour, Senior Member, IEEE, and Gholamreza Moradi, Member, IEEE, "Bandwidth and Gain Enhancement of an Aperture Antenna with Modified Ring Patch" IEEE ANTENNAS AND WIRELESS PROPAGATION LETTERS, VOL. 10 ,

[2] Yikai Chen, Shiwen Yang, and Zaiping Nie , Bandwidth Enhancement Method for Low Profile E-Shaped Micro strip Patch Antennas", IEEE TRANSACTIONS ON ANTENNAS AND PROPAGATION, VOL. 58, NO. 7, JULY 2010.
[3] H. F. AbuTarboush, H. S. Al-Raweshidy and R. Nilavalan," Bandwidth Enhancement for Small Patch Antenna Using PBG Structure for Different Wireless Applications" IEEE TRANSACTIONS ON ANTENNAS AND PROPAGATION, 2009

[4] D. M. Pozar, "Micro strip antennas", Proceedings of the IEEE. Volume 80, Issue 1, January 1992, pp $79-91$.

[5] C. A. Balanis, "Antenna Theory: Analysis and Design", 2nd Ed. New York: John Wiley and Sons. 1997 pp. $720-784$

[6] Thomas A. Milligan "2nd addition Modern antenna design”, pp: 318-354

[7] Keith R. Carverand James W. Mink, “ Microstrip Antenna Technology", IEEE Trans.Antenna Propagation, vol. AP-29, no. 1, Jan.1981 pp. 2-24.

[8] M.A.A, S.H, Ahmad. A. K. and Jama S.M, "Cavity model analysis of rectangular microstrip antenna operating in TM03 mode", IEEE proceeding, Feb 2006, pp.2218-2223.

[9] A. F. Tinoco S., D.C. Nascimento, and J. C. Da S. Lacava, "Rectangular Microstrip Antenna Design Suitable for Undergraduate courses", IEEE Proceeding, Jan 2008.

[10] K. George Thomas, M. Sreenivasan, "A simple dual-band microstrip-fed printed antenna for WLAN applications", IET Microwave Antennas Propagation, Vol. 3, Iss. 4, 2009, pp.687-694.

[11] Aditi Sharma, G. Singh and D. S. Chauhan, "Design Considerations to Improve the Performance of a Rectangular Microstrip Patch Antenna at THz Frequency", IEEE proceeding, Jun 2008, pp. 1-2.

[12] M. Rahman, M. Stuchly, "Modeling and Application of 2D Photonic Band Gap Structure", IEEE Proc. MTT-S, Vol. 2, March 2001.

[13] Bin Lin, Baiqiang You, Jianhua Zhou, "The Microstrip Antenna with PBG Used for 3G system”, IEEE Proc. July 2007.

[14] Y.J. Sung and Y.S. Kim. "An Improved Design of Microstrip Patch Antennas Using Photonic Bandgap Structure", IEEE Transactions on Antennas and Propagation, Vol. 53, No.5, May 2005. 